\title{
A nonautonomous impulsive stochastic population model with nonlinear interspecific competitive terms
}

\author{
Ronghua Tan ${ }^{1 *}$, Shengliang Guo ${ }^{1}$, Lianwen Wang ${ }^{1}$, Dashun $\mathrm{Xu}^{1,2}$ and Zhijun Liu ${ }^{1}$
}

\section{"Correspondence:}

ronghua_tan@hotmail.com

'Department of Mathematics,

Hubei University for Nationalities,

Enshi, P.R. China

Full list of author information is

available at the end of the article

\section{Springer}

\begin{abstract}
In this paper, we formulate an nonautonomous impulsive population model with nonlinear interspecific competitive terms, and introduce the random perturbation of the birth rates of two species into this model. A good understanding of the extinction, stochastic permanence and global attractivity of system is obtained. Also, the limit of the average in time of the sample paths of every component of solutions is estimated. Numerical simulations are performed to justify our analytical results.
\end{abstract}

Keywords: Randomized competitive system; Impulse; Asymptotic behaviors

\section{Introduction}

Recently, Liu et al. [1] proposed the following nonautonomous two-species competitive system with impulsive perturbations:

$$
\left\{\begin{array}{l}
d x_{1}(t)=x_{1}(t)\left[a_{1}(t)-b_{1}(t) x_{1}(t)-\frac{c_{2}(t) x_{2}(t)}{1+x_{2}(t)}\right] d t, \\
d x_{2}(t)=x_{2}(t)\left[a_{2}(t)-b_{2}(t) x_{2}(t)-\frac{c_{1}(t) x_{1}(t)}{1+x_{1}(t)}\right] d t,
\end{array}\right\} \quad t \neq t_{k},
$$

where the parameters $a_{i}(t), b_{i}(t)$ and $c_{i}(t)$ are positive continuous bounded functions on $[0,+\infty)$, and stand up the intrinsic growth rates, intraspecific competitive rates and interspecific competitive rates, respectively. $\mathbb{N}$ is the set of positive integers. $t_{k}$ are impulsive points satisfying $0<t_{1}<t_{2}<\cdots, \lim _{k \rightarrow+\infty} t_{k}=+\infty, k \in \mathbb{N}$, and $\mathcal{H}_{i k}>-1, i=1,2$. There exist positive constants $h_{i}$ and $H_{i}$ such that $h_{i} \leq \prod_{0<t_{k}<t}\left(1+\mathcal{H}_{i k}\right) \leq H_{i}$.

Note that May [2] revealed the fact that due to environmental noises, the birth rate, carrying capacity, competition coefficient and other parameters involved in the system exhibit random fluctuation to a greater or lesser extent. Based on the above basic assumption, it is reasonable to introduce the environment noise into system (1.1). For more precisely, we estimate the birth rate of each species by an average value plus errors which follow a normal distribution. That is, we may replace the parameters $a_{i}(t)$ by $a_{i}(t)+\sigma_{i}(t) \dot{B}_{i}(t)$, respectively. Here $\dot{B}_{i}(t)$ are white noises, and $B_{i}(t)$ are the independent standard Brownian motions defined on a complete probability space $\left(\Omega, \mathcal{F},\{\mathcal{F}\}_{t \geq 0}, \mathbb{P}\right)$ with a filtration $\{\mathcal{F}\}_{t \geq 0}$

(c) The Author(s) 2018. This article is distributed under the terms of the Creative Commons Attribution 4.0 International License (http://creativecommons.org/licenses/by/4.0/), which permits unrestricted use, distribution, and reproduction in any medium, provided you give appropriate credit to the original author(s) and the source, provide a link to the Creative Commons license, and indicate if changes were made. 
satisfying the usual conditions. $\sigma_{i}^{2}(t)$ represent the intensities of the noises and $\sigma_{i}(t)$ are positive continuous bounded functions on $[0,+\infty)$. Then we obtain the following impulsive stochastic model:

$$
\left\{\begin{array}{l}
d x_{1}(t)=x_{1}(t)\left[a_{1}(t)-b_{1}(t) x_{1}(t)-\frac{c_{2}(t) x_{2}(t)}{1+x_{2}(t)}\right] d t+\sigma_{1}(t) x_{1}(t) d B_{1}(t), \\
d x_{2}(t)=x_{2}(t)\left[a_{2}(t)-b_{2}(t) x_{2}(t)-\frac{c_{1}(t) x_{1}(t)}{1+x_{1}(t)}\right] d t+\sigma_{2}(t) x_{2}(t) d B_{2}(t),
\end{array}\right\} \quad t \neq t_{k},
$$

The main aims of this contribution are to investigate the extinction, stochastic permanence, the limit of the average in time of the sample paths of solutions and global attractivity, which is a continuation of previous work $[1,3,4]$.

To proceed, let us give the following symbols:

- For a continuous and bounded function $f(t)$, let $f^{l}=\inf _{t \geq 0} f(t), f^{u}=\sup _{t \geq 0} f(t)$.

- For any constant sequence $\left\{\varsigma_{i}\right\}, 1 \leq i, j \leq 2$, let $\hat{\zeta}=\min _{1 \leq i \leq 2} \varsigma_{i}, \check{\zeta}=\max _{1 \leq i \leq 2} \varsigma_{i}$.

The following definitions are commonly used.

\section{Definition 1.1}

- System (1.2) is said to be extinctive if for initial value $x(0)>0$, the solution $x(t)$ satisfies $\lim _{t \rightarrow+\infty} x(t)=0$ a.s.

- System (1.2) is said to be stochastically permanent if for every $\varepsilon \in(0,1)$, there exists a pair of positive constants $\alpha, \beta$ such that, for initial value $x(0)>0$, the solution $x(t)$ satisfies

$$
\liminf _{t \rightarrow+\infty} \mathbb{P}\{x(t) \geq \alpha\} \geq 1-\varepsilon, \quad \liminf _{t \rightarrow+\infty} \mathbb{P}\{x(t) \leq \beta\} \geq 1-\varepsilon .
$$

Definition 1.2 Let $x(t), x^{*}(t)$ be any two solutions of system (1.2) with initial values $x(0)>0, x^{*}(0)>0$, respectively. If $\lim _{t \rightarrow+\infty}\left|x(t)-x^{*}(t)\right|=0$ a.s., then system (1.2) is globally attractive.

The remaining parts of the paper is organized as follows. Section 2 is devoted to a discussion of the existence and positivity of solutions of system (1.2). In Sect. 3, the extinction and stochastic permanence of system (1.2) are investigated. In Sect. 4, we verify that the limit of the average in time of the sample paths of every component of the solution is bounded and an estimation for it is also given. Section 5 establishes the global attractivity of system (1.2). In Sects. 6 and 7, numerical simulations and conclusions are presented, respectively.

\section{Global positive solutions}

In order to study system (1.2), the following non-impulsive system is always needed:

$$
\left\{\begin{aligned}
d y_{1}(t)= & y_{1}(t)\left[a_{1}(t)-b_{1}(t) \prod_{0<t_{k}<t}\left(1+\mathcal{H}_{1 k}\right) y_{1}(t)-\frac{c_{2}(t) \prod_{0<t_{k}<t}\left(1+\mathcal{H}_{2 k}\right) y_{2}(t)}{1+\prod_{0<t_{k}<t}\left(1+\mathcal{H}_{2 k}\right) y_{2}(t)}\right] d t \\
& +\sigma_{1}(t) y_{1}(t) d B_{1}(t), \\
d y_{2}(t)= & y_{2}(t)\left[a_{2}(t)-b_{2}(t) \prod_{0<t_{k}<t}\left(1+\mathcal{H}_{2 k}\right) y_{2}(t)-\frac{c_{1}(t) \prod_{0<t_{k}<t}\left(1+\mathcal{H}_{1 k}\right) y_{1}(t)}{1+\prod_{0<t_{k}<t}\left(1+\mathcal{H}_{1 k}\right) y_{1}(t)}\right] d t \\
& +\sigma_{2}(t) y_{2}(t) d B_{2}(t),
\end{aligned}\right.
$$

with initial value $y(0)=x(0)>0$. 
Theorem 2.1 For initial condition $x(0)>0$, there is a unique solution $x(t)$ to system (1.2) for all $t \geq 0$ and $x(t)$ will remain in $R_{+}^{2}$ with probability one.

Proof The method of our proof is similar to that of Ref. [5]. It follows from the theory of stochastic differential equations (see [6]) that system (2.1) admits a unique continuous maximal local solution $\left(y_{1}(t), y_{2}(t)\right)$ on $\left[0, \tau_{*}\right)$, where $\tau_{*}$ is the explosion time. To show the solution is global, we need to show that $\tau_{*}=+\infty$ a.s. Let $m_{0}$ be sufficiently large for every component of $\left(y_{1}(0), y_{2}(0)\right)$ lying within the interval $\left[\frac{1}{m_{0}}, m_{0}\right]$. For each integer $m \geq m_{0}$, define the stopping time

$$
\tau_{m}=\inf \left\{t \in\left[0, \tau_{*}\right): y_{1}(t) \notin\left(\frac{1}{m}, m\right) \text { or } y_{2}(t) \notin\left(\frac{1}{m}, m\right)\right\} .
$$

Then $\tau_{m}$ is increasing as $m$ increases. Set $\tau_{+\infty}=\lim _{m \rightarrow+\infty} \tau_{m}$, whence $\tau_{+\infty} \leq \tau_{*}$ a.s. If one can show that $\tau_{+\infty}=+\infty$ a.s., then $\tau_{*}=+\infty$ a.s. If not, then there is a pair of constants $T>0$ and $\varepsilon \in(0,1)$ such that $\mathbb{P}\left\{\tau_{+\infty} \leq T\right\}>\varepsilon$. Hence there is an integer $m_{1} \geq m_{0}$ such that, for all $m \geq m_{1}, \mathbb{P}\left\{\tau_{m} \leq T\right\} \geq \varepsilon$.

Assign a function $V: R_{+}^{2} \rightarrow R_{+}$with the form

$$
V(y)=y_{1}-1-\ln y_{1}+y_{2}-1-\ln y_{2} .
$$

Then the nonnegativity of this function can be deduced from $s-1-\ln s \geq 0$ on $s>0$. Applying Itô's formula, one has

$$
\begin{aligned}
d V\left(y_{1}, y_{2}\right)= & \left(1-\frac{1}{y_{1}}\right) d y_{1}+0.5 \frac{1}{y_{1}^{2}}\left(d y_{1}\right)^{2}+\left(1-\frac{1}{y_{2}}\right) d y_{2}+0.5 \frac{1}{y_{2}^{2}}\left(d y_{2}\right)^{2} \\
= & \left\{( y _ { 1 } ( t ) - 1 ) \left[a_{1}(t)-b_{1}(t) \prod_{0<t_{k}<t}\left(1+\mathcal{H}_{1 k}\right) y_{1}(t)\right.\right. \\
& \left.\left.-\frac{c_{2}(t) \prod_{0<t_{k}<t}\left(1+\mathcal{H}_{2 k}\right) y_{2}(t)}{1+\prod_{0<t_{k}<t}\left(1+\mathcal{H}_{2 k}\right) y_{2}(t)}\right]+0.5 \sigma_{1}^{2}(t)\right\} d t+\left(y_{1}(t)-1\right) \sigma_{1}(t) d B_{1}(t) \\
& +\left\{( y _ { 2 } ( t ) - 1 ) \left[a_{2}(t)-b_{2}(t) \prod_{0<t_{k}<t}\left(1+\mathcal{H}_{2 k}\right) y_{2}(t)\right.\right. \\
& \left.\left.-\frac{c_{1}(t) \prod_{0<t_{k}<t}\left(1+\mathcal{H}_{1 k}\right) y_{1}(t)}{1+\prod_{0<t_{k}<t}\left(1+\mathcal{H}_{1 k}\right) y_{1}(t)}\right]+0.5 \sigma_{2}^{2}(t)\right\} d t+\left(y_{2}(t)-1\right) \sigma_{2}(t) d B_{2}(t) \\
= & \left\{a_{1}(t) y_{1}(t)-b_{1}(t) \prod_{0<t_{k}<t}\left(1+\mathcal{H}_{1 k}\right) y_{1}^{2}(t)-\frac{c_{2}(t) \prod_{0<t_{k}<t}\left(1+\mathcal{H}_{2 k}\right) y_{1}(t) y_{2}(t)}{1+\prod_{0<t_{k}<t}\left(1+\mathcal{H}_{2 k}\right) y_{2}(t)}\right. \\
& -a_{1}(t)+b_{1}(t) \prod_{0<t_{k}<t}\left(1+\mathcal{H}_{1 k}\right) y_{1}(t) \\
& +\frac{c_{2}(t) \prod_{0<t_{k}<t}\left(1+\mathcal{H}_{2 k}\right) y_{2}(t)}{1+\prod_{0<t_{k}<t}\left(1+\mathcal{H}_{2 k}\right) y_{2}(t)}+0.5 \sigma_{1}^{2}(t) \\
& +a_{2}(t) y_{2}(t)-b_{2}(t) \prod_{0<t_{k}<t}\left(1+\mathcal{H}_{2 k}\right) y_{2}^{2}(t)-\frac{c_{1}(t) \prod_{0<t_{k}<t}\left(1+\mathcal{H}_{1 k}\right) y_{1}(t) y_{2}(t)}{1+\prod_{0<t_{k}<t}\left(1+\mathcal{H}_{1 k}\right) y_{1}(t)}
\end{aligned}
$$




$$
\begin{aligned}
& -a_{2}(t)+b_{2}(t) \prod_{0<t_{k}<t}\left(1+\mathcal{H}_{2 k}\right) y_{2}(t)+\frac{c_{1}(t) \prod_{0<t_{k}<t}\left(1+\mathcal{H}_{1 k}\right) y_{1}(t)}{1+\prod_{0<t_{k}<t}\left(1+\mathcal{H}_{1 k}\right) y_{1}(t)} \\
& \left.+0.5 \sigma_{2}^{2}(t)\right\} d t+\left(y_{1}(t)-1\right) \sigma_{1}(t) d B_{1}(t)+\left(y_{2}(t)-1\right) \sigma_{2}(t) d B_{2}(t) \\
\leq & \left\{-b_{1}(t) \prod_{0<t_{k}<t}\left(1+\mathcal{H}_{1 k}\right) y_{1}^{2}(t)+\left(a_{1}(t)+b_{1}(t) \prod_{0<t_{k}<t}\left(1+\mathcal{H}_{1 k}\right)\right) y_{1}(t)\right. \\
& +c_{2} \prod_{0<t_{k}<t}\left(1+\mathcal{H}_{2 k}\right) y_{2}(t)-a_{1}(t)+0.5 \sigma_{1}^{2}(t)-b_{2}(t) \prod_{0<t_{k}<t}\left(1+\mathcal{H}_{2 k}\right) y_{2}^{2}(t) \\
& +\left(a_{2}(t)+b_{2}(t) \prod_{0<t_{k}<t}\left(1+\mathcal{H}_{2 k}\right)\right) y_{2}(t)+c_{1} \prod_{0<t_{k}<t}\left(1+\mathcal{H}_{1 k}\right) y_{1}(t)-a_{2}(t) \\
& \left.+0.5 \sigma_{2}^{2}(t)\right\} d t+\left(y_{1}(t)-1\right) \sigma_{1}(t) d B_{1}(t)+\left(y_{2}(t)-1\right) \sigma_{2}(t) d B_{2}(t) \\
\leq & \left\{-b_{1}^{l} h_{1} y_{1}^{2}(t)+\left(a_{1}^{u}+b_{1}^{u} H_{1}+c_{1}^{u} H_{1}\right) y_{1}(t)-a_{1}^{l}+0.5\left(\sigma_{1}^{u}\right)^{2}-b_{2}^{l} h_{2} y_{2}^{2}(t)\right. \\
& \left.+\left(a_{2}^{u}+b_{2}^{u} H_{2}+c_{2}^{u} H_{2}\right) y_{2}(t)-a_{2}^{l}+0.5\left(\sigma_{2}^{u}\right)^{2}\right\} d t \\
& +\left(y_{1}(t)-1\right) \sigma_{1}(t) d B_{1}(t)+\left(y_{2}(t)-1\right) \sigma_{2}(t) d B_{2}(t) \\
= & \Delta\left(y_{1}(t), y_{2}(t)\right) d t+\left(y_{1}(t)-1\right) \sigma_{1} d B_{1}(t)+\left(y_{2}(t)-1\right) \sigma_{2} d B_{2}(t),
\end{aligned}
$$

where

$$
\begin{aligned}
\Delta\left(y_{1}, y_{2}\right)= & -b_{1}^{l} h_{1} y_{1}^{2}(t)+\left(a_{1}^{u}+b_{1}^{u} H_{1}+c_{1}^{u} H_{1}\right) y_{1}(t)-a_{1}^{l}+0.5\left(\sigma_{1}^{u}\right)^{2}-b_{2}^{l} h_{2} y_{2}^{2}(t) \\
& +\left(a_{2}^{u}+b_{2}^{u} H_{2}+c_{2}^{u} H_{2}\right) y_{2}(t)-a_{2}^{l}+0.5\left(\sigma_{2}^{u}\right)^{2} .
\end{aligned}
$$

A calculation can show that $\Delta\left(y_{1}(t), y_{2}(t)\right)$ is upper bounded, denoted by $\mathscr{K}$. The rest of proof is similar to Theorem 2.1 in Ref. [7]; we omit it.

Now let

$$
x_{1}(t)=\prod_{0<t_{k}<t}\left(1+\mathcal{H}_{1 k}\right) y_{1}(t), \quad x_{2}(t)=\prod_{0<t_{k}<t}\left(1+\mathcal{H}_{2 k}\right) y_{2}(t) .
$$

In what follows, it will be shown that $x(t)=\left(x_{1}(t), x_{2}(t)\right)$ is the solution of system (1.2). We first prove that $x_{1}(t)$ satisfies system (1.2). In fact, $x_{1}(t)$ is continuous on $\left(0, t_{1}\right)$ and each interval $\left(t_{k}, t_{k+1}\right) \subset[0,+\infty)$, and for $t \neq t_{k}$,

$$
\begin{aligned}
d x_{1}(t)= & d\left(\prod_{0<t_{k}<t}\left(1+\mathcal{H}_{1 k}\right) y_{1}(t)\right)=\prod_{0<t_{k}<t}\left(1+\mathcal{H}_{1 k}\right) d y_{1}(t) \\
= & \prod_{0<t_{k}<t}\left(1+\mathcal{H}_{1 k}\right) y_{1}(t)\left[a_{1}(t)-b_{1}(t) \prod_{0<t_{k}<t}\left(1+\mathcal{H}_{1 k}\right) y_{1}(t)\right. \\
& \left.-\frac{c_{2}(t) \prod_{0<t_{k}<t}\left(1+\mathcal{H}_{2 k}\right) y_{2}(t)}{1+\prod_{0<t_{k}<t}\left(1+\mathcal{H}_{2 k}\right) y_{2}(t)}\right] d t+\prod_{0<t_{k}<t}\left(1+\mathcal{H}_{1 k}\right) \sigma_{1}(t) y_{1}(t) d B_{1}(t) \\
= & x_{1}(t)\left[a_{1}(t)-b_{1}(t) x_{1}(t)-\frac{c_{2}(t) x_{2}(t)}{1+x_{2}(t)}\right] d t+\sigma_{1}(t) x_{1}(t) d B_{1}(t) .
\end{aligned}
$$


Meanwhile, for every $k \in \mathbb{N}$ and $t_{k} \in[0,+\infty)$, one has

$$
\begin{aligned}
x_{1}\left(t_{k}^{+}\right) & =\lim _{t \rightarrow t_{k}^{+}} \prod_{0<t_{j}<t}\left(1+\mathcal{H}_{1 k}\right) y_{1}(t)=\prod_{0<t_{j} \leq t_{k}}\left(1+\mathcal{H}_{1 k}\right) y_{1}\left(t_{k}^{+}\right) \\
& =\left(1+\mathcal{H}_{1 k}\right) \prod_{0<t_{j}<t_{k}}\left(1+\mathcal{H}_{1 k}\right) y_{1}\left(t_{k}\right)=\left(1+\mathcal{H}_{1 k}\right) x_{1}\left(t_{k}\right)
\end{aligned}
$$

and

$$
\begin{aligned}
x_{1}\left(t_{k}^{-}\right) & =\lim _{t \rightarrow t_{k}^{-}} \prod_{0<t_{j}<t}\left(1+\mathcal{H}_{1 k}\right) y_{1}(t)=\prod_{0<t_{j}<t_{k}}\left(1+\mathcal{H}_{1 k}\right) y_{1}\left(t_{k}^{-}\right) \\
& =\prod_{0<t_{j}<t_{k}}\left(1+\mathcal{H}_{1 k}\right) y_{1}\left(t_{k}\right)=x_{1}\left(t_{k}\right) .
\end{aligned}
$$

Similarly, it can be verified that $x_{2}(t)$ satisfies system (1.2). By the definition of a solution of an impulsive stochastic differential equation (ISDE) (see [5]), we find that $x(t)$ is a solution of system (1.2). The proof of Theorem 2.1 is complete.

\section{Extinction and stochastic permanence}

Assign

$$
\mathcal{R}_{i}(t)=a_{i}(t)-0.5 \sigma_{i}^{2}(t), \quad i=1,2,
$$

and

$$
\mathcal{R}_{i}^{*}=\limsup _{t \rightarrow+\infty} t^{-1}\left(\sum_{0<t_{k}<t} \ln \left(1+\mathcal{H}_{i k}\right)+\int_{0}^{t} \mathcal{R}_{i}(s) d s\right) .
$$

The following theorem shows that both species go to extinction if the noise intensities are sufficiently large.

Theorem 3.1 The solution $x(t)$ of system (1.2) satisfies $\limsup _{t \rightarrow+\infty} t^{-1} \ln x_{i}(t) \leq \mathcal{R}_{i}^{*}$ a.s. In particular, if $\mathcal{R}_{i}^{*}<0$, then $\lim _{t \rightarrow+\infty} x_{i}(t)=0$ a.s.

Proof By Itô’s formula, one can deduce from (2.1) that

$$
\begin{aligned}
d \ln y_{i}(t)= & \left(a_{i}(t)-b_{i}(t) \prod_{0<t_{k}<t}\left(1+\mathcal{H}_{i k}\right) y_{i}(t)-\frac{c_{j}(t) \prod_{0<t_{k}<t}\left(1+\mathcal{H}_{j k}\right) y_{j}(t)}{1+\prod_{0<t_{k}<t}\left(1+\mathcal{H}_{j k}\right) y_{j}(t)}\right. \\
& \left.-0.5 \sigma_{i}^{2}(t)\right) d t+\sigma_{i}(t) d B_{i}(t) .
\end{aligned}
$$

Hence

$$
\begin{aligned}
\ln y_{i}(t)-\ln y_{i}(0)= & \int_{0}^{t} \mathcal{R}_{i}(s) d s-\int_{0}^{t} b_{i}(s) \prod_{0<t_{k}<s}\left(1+\mathcal{H}_{i k}\right) y_{i}(s) d s \\
& -\int_{0}^{t} \frac{c_{j}(s) \prod_{0<t_{k}<s}\left(1+\mathcal{H}_{j k}\right) y_{j}(s)}{1+\prod_{0<t_{k}<s}\left(1+\mathcal{H}_{j k}\right) y_{j}(s)} d s+\mathscr{M}_{i}(t),
\end{aligned}
$$


where

$$
\mathscr{M}_{i}(t)=\int_{0}^{t} \sigma_{i}(s) d B_{i}(s)
$$

and the quadratic variation of this martingale takes the form

$$
\left\langle\mathscr{M}_{i}(t), \mathscr{M}_{i}(t)\right\rangle=\int_{0}^{t} \sigma_{i}^{2}(s) d s \leq\left(\sigma_{i}^{u}\right)^{2} t .
$$

By the strong law of large numbers for local martingale (see [6]), it can be seen that

$$
\lim _{t \rightarrow+\infty} \frac{\mathscr{M}_{i}(t)}{t}=0 \quad \text { a.s. }
$$

From (3.4) one has

$$
\begin{aligned}
& \sum_{0<t_{k}<t} \ln \left(1+\mathcal{H}_{i k}\right)+\ln y_{i}(t)-\ln y_{i}(0) \\
& =\sum_{0<t_{k}<t} \ln \left(1+\mathcal{H}_{i k}\right)+\int_{0}^{t} \mathcal{R}_{i}(s) d s-\int_{0}^{t} b_{i}(s) \prod_{0<t_{k}<s}\left(1+\mathcal{H}_{i k}\right) y_{i}(s) d s \\
& \quad-\int_{0}^{t} \frac{c_{j}(s) \prod_{0<t_{k}<s}\left(1+\mathcal{H}_{j k}\right) y_{j}(s)}{1+\prod_{0<t_{k}<s}\left(1+\mathcal{H}_{j k}\right) y_{j}(s)} d s+\mathscr{M}_{i}(t) .
\end{aligned}
$$

Consequently,

$$
\ln x_{i}(t)-\ln x_{i}(0) \leq \sum_{0<t_{k}<t} \ln \left(1+\mathcal{H}_{i k}\right)+\int_{0}^{t} \mathcal{R}_{i}(s) d s+\mathscr{M}_{i}(t) .
$$

And thus the required assertion follows from (3.7). The proof of Theorem 3.1 is finished.

In contrast with Theorem 3.1, two species can coexist. Theorem 3.2 shows that system (1.2) is stochastically permanent provided that the noise intensity is suitably small. Let

$$
\hat{\mathcal{R}}^{l}=\min \left\{\inf _{t \geq 0} \mathcal{R}_{1}(t), \inf _{t \geq 0} \mathcal{R}_{2}(t)\right\} .
$$

Theorem 3.2 If $\hat{\mathcal{R}}^{l}>0$, then system (1.2) is stochastically permanent.

Proof We first define

$$
V_{1}(y)=\frac{1}{W^{2}}, \quad W=y_{1}+y_{2}, y \in R_{+}^{2} .
$$

An application of Itô’s formula yields

$$
\begin{aligned}
d V_{1}(y) & =-\frac{2}{W^{3}} d W+\frac{3}{W^{4}}(d W)^{2} \\
& =-\frac{2}{W^{3}}\left\{y_{1}(t)\left[a_{1}(t)-b_{1}(t) \prod_{0<t_{k}<t}\left(1+\mathcal{H}_{1 k}\right) y_{1}(t)-\frac{c_{2}(t) \prod_{0<t_{k}<t}\left(1+\mathcal{H}_{2 k}\right) y_{2}(t)}{1+\prod_{0<t_{k}<t}\left(1+\mathcal{H}_{2 k}\right) y_{2}(t)}\right]\right.
\end{aligned}
$$




$$
\begin{aligned}
& \left.+y_{2}(t)\left[a_{2}(t)-b_{2}(t) \prod_{0<t_{k}<t}\left(1+\mathcal{H}_{2 k}\right) y_{2}(t)-\frac{c_{1}(t) \prod_{0<t_{k}<t}\left(1+\lambda_{1 k}\right) y_{1}(t)}{1+\prod_{0<t_{k}<t}\left(1+\mathcal{H}_{1 k}\right) y_{1}(t)}\right]\right\} d t \\
& +\frac{3}{W^{4}}\left(\sigma_{1}^{2}(t) y_{1}^{2}(t)+\sigma_{2}^{2}(t) y_{2}^{2}(t)\right) d t \\
& -\frac{2}{W^{3}}\left(\sigma_{1}(t) y_{1}(t) d B_{1}(t)+\sigma_{2}(t) y_{2}(t) d B_{2}(t)\right) .
\end{aligned}
$$

It follows from $\hat{\mathcal{R}}^{l}>0$ that one can choose a constant $\varrho>0$ such that

$$
\hat{\mathcal{R}}^{l}>\varrho\left(\check{\sigma}^{u}\right)^{2}
$$

Assign

$$
V_{2}(y)=\left(1+V_{1}(y)\right)^{\varrho}
$$

One deduces from Itô’s formula that

$$
\begin{aligned}
& d V_{2}(y)= \varrho\left(1+V_{1}(y)\right)^{\varrho-1} d V_{1}(y)+0.5 \varrho(\varrho-1)\left(1+V_{1}(y)\right)^{\varrho-2}\left(d V_{1}(y)\right)^{2} \\
&= \varrho\left(1+V_{1}(y)\right)^{\varrho-2}\left\{-\left(1+V_{1}(y)\right) \frac{2}{W^{3}}\left[a_{1}(t) y_{1}(t)-b_{1}(t) \prod_{0<t_{k}<t}\left(1+\mathcal{H}_{1 k}\right) y_{1}^{2}(t)\right.\right. \\
&-\frac{c_{2}(t) \prod_{0<t_{k}<t}\left(1+\mathcal{H}_{2 k}\right) y_{1}(t) y_{2}(t)}{1+\prod_{0<t_{k}<t}\left(1+\mathcal{H}_{2 k}\right) y_{2}(t)}+a_{2}(t) y_{2}(t)-b_{2}(t) \prod_{0<t_{k}<t}\left(1+\mathcal{H}_{2 k}\right) y_{2}^{2}(t) \\
&\left.-\frac{c_{1}(t) \prod_{0<t_{k}<t}\left(1+\mathcal{H}_{1 k}\right) y_{1}(t) y_{2}(t)}{1+\prod_{0<t_{k}<t}\left(1+\mathcal{H}_{1 k}\right) y_{1}(t)}\right]+\left(\frac{3}{W^{4}}+\frac{3}{W^{6}}+\frac{2 \varrho-2}{W^{6}}\right)\left(\sigma_{1}^{2}(t) y_{1}^{2}(t)\right. \\
&\left.\left.+\sigma_{2}^{2}(t) y_{2}^{2}(t)\right)\right\} d t-\varrho\left(1+V_{1}(y)\right)^{\varrho-1} \frac{2}{W^{3}}\left(\sigma_{1}(t) y_{1}(t) d B_{1}(t)+\sigma_{2}(t) y_{2}(t) d B_{2}(t)\right) \\
& \leq \varrho\left(1+V_{1}(y)\right)^{\varrho-2}\left\{-\frac{2}{W^{5}}\left[a_{1}(t) y_{1}(t)+a_{2}(t) y_{2}(t)\right]\right. \\
&+\frac{2}{W^{3}}\left[b_{1}(t) \prod_{0<t_{k}<t}\left(1+\mathcal{H}_{1 k}\right) y_{1}^{2}(t)+\frac{c_{2}(t) \prod_{0<t_{k}<t}\left(1+\mathcal{H}_{2 k}\right) y_{1}(t) y_{2}(t)}{1+\prod_{0<t_{k}<t}\left(1+\mathcal{H}_{2 k}\right) y_{2}(t)}\right. \\
&\left.+\frac{c_{1}(t) \prod \prod_{0<t_{k}<t}\left(1+\mathcal{H}_{1 k}\right) y_{1}(t) y_{2}(t)}{1+\prod_{0<t_{k}<t}\left(1+\mathcal{H}_{1 k}\right) y_{1}(t)}+b_{2}(t) \prod_{0<t_{k}<t}\left(1+\mathcal{H}_{2 k}\right) y_{2}^{2}(t)\right] \\
& \leq \varrho\left(1+V_{1}(y)\right)^{\varrho-2}\left\{-\frac{2}{W^{6}}\left[y_{1}^{2}(t)\left(a_{1}(t)-0.5 \sigma_{1}^{2}(t)-\varrho \sigma_{1}^{2}(t)\right)\right.\right. \\
&+\frac{2}{W^{5}}\left[b_{1}(t) \prod_{0<t_{k}<t}\left(1+\mathcal{H}_{1 k}\right) y_{1}^{2}(t)+\frac{c_{2}(t) \prod_{0<t_{k}<t}\left(1+\mathcal{H}_{2 k}\right) y_{1}(t) y_{2}(t)}{1+\prod_{0<t_{k}<t}\left(1+\mathcal{H}_{2 k}\right) y_{2}(t)}\right. \\
&\left.+\frac{c_{1}(t) \prod \prod_{0<t_{k}<t}\left(1+\mathcal{H}_{1 k}\right) y_{1}(t) y_{2}(t)}{1+\prod_{0<t_{k}<t}\left(1+\mathcal{H}_{1 k}\right) y_{1}(t)}+b_{2}(t) \prod_{0<t_{k}<t}\left(1+\mathcal{H}_{2 k}\right) y_{2}^{2}(t)\right] \\
&\left.\left.+\frac{3}{W^{4}}+\frac{2 \varrho+1}{W^{6}}\right)\left(\sigma_{1}^{2}(t) y_{1}^{2}(t)+\sigma_{2}^{2}(t) y_{2}^{2}(t)\right)\right\} d t \\
&\left.W_{1}(y)\right)^{\varrho-1} \frac{2}{W^{3}}\left(\sigma_{1}(t) y_{1}(t) d B_{1}(t)+\sigma_{2}(t) y_{2}(t) d B_{2}(t)\right) \\
&
\end{aligned}
$$




$$
\begin{aligned}
& \left.+y_{2}^{2}(t)\left(a_{2}(t)-0.5 \sigma_{2}^{2}(t)-\varrho \sigma_{2}^{2}(t)\right)\right] \\
& \left.+\frac{2 H \check{b}^{u}}{W}+\frac{2 H \check{c}^{u}}{W}+\frac{2 H \check{b}^{u}}{W^{3}}+\frac{2 H \check{c}^{u}}{W^{3}}+\frac{3\left(\check{\sigma}^{u}\right)^{2}}{W^{2}}\right\} d t \\
& -\varrho\left(1+V_{1}(y)\right)^{\varrho-1} \frac{2}{W^{3}}\left(\sigma_{1}(t) y_{1}(t) d B_{1}(t)+\sigma_{2}(t) y_{2}(t) d B_{2}(t)\right) \\
\leq & \varrho\left(1+V_{1}(y)\right)^{\varrho-2}\left\{-\frac{1}{2}\left(\hat{\mathcal{R}}^{l}-\varrho\left(\check{\sigma}^{u}\right)^{2}\right) V_{1}^{2}(y)+\left(2 H \check{b}^{u}+2 H \check{c}^{u}\right) V_{1}^{0.5}(y)\right. \\
& \left.+\left(2 H \check{b}^{u}+2 H \check{c}^{u}\right) V_{1}^{1.5}(y)+3\left(\check{\sigma}^{u}\right)^{2} V_{1}(y)\right\} d t \\
& -\varrho\left(1+V_{1}(y)\right)^{\varrho-1} \frac{2}{W^{3}}\left(\sigma_{1}(t) y_{1}(t) d B_{1}(t)+\sigma_{2}(t) y_{2}(t) d B_{2}(t)\right),
\end{aligned}
$$

where $H=\max \left\{H_{1}, H_{2}\right\}, H_{i}$ is defined in (1.1). By (3.11), we can choose a sufficiently small $\kappa$ satisfying

$$
\hat{\mathcal{R}}^{l}-\varrho\left(\check{\sigma}^{u}\right)^{2}>\frac{2 \kappa}{\varrho}>0
$$

Assign

$$
V_{3}(y)=e^{\kappa t} V_{2}(y) .
$$

By application of Itô's formula, one finds that

$$
\begin{aligned}
d V_{3}(y)= & \kappa e^{\kappa t} V_{2}(y) d t+e^{\kappa t} d V_{2}(y) \\
\leq & \varrho e^{\kappa t}\left(1+V_{1}(y)\right)^{\varrho-2}\left\{\frac{\kappa}{\varrho}\left(1+V_{1}(y)\right)^{2}-\frac{1}{2}\left(\hat{\mathcal{R}}^{l}-\varrho\left(\check{\sigma}^{u}\right)^{2}\right) V_{1}^{2}(y)\right. \\
& \left.+3\left(\check{\sigma}^{u}\right)^{2} V_{1}(y)+\left(2 H \check{b}^{u}+2 H \check{c}^{u}\right) V_{1}^{0.5}(y)+\left(2 H \check{b}^{u}+2 H \check{c}^{u}\right) V_{1}^{1.5}(y)\right\} d t \\
& -\varrho e^{\kappa t}\left(1+V_{1}(y)\right)^{\varrho-1} \frac{2}{W^{3}}\left(\sigma_{1}(t) y_{1}(t) d B_{1}(t)+\sigma_{2}(t) y_{2}(t) d B_{2}(t)\right) \\
= & \varrho e^{\kappa t}\left(1+V_{1}(y)\right)^{\varrho-2}\left\{-2 V_{1}^{2}(y)\left[\frac{\hat{\mathcal{R}}^{l}-\varrho\left(\check{\sigma}^{u}\right)^{2}}{4}-\frac{0.5 \kappa}{\varrho}\right]\right. \\
& +\left[3\left(\check{\sigma}^{u}\right)^{2}+\frac{2 \kappa}{\varrho}\right] V_{1}(y)+\left(2 H \check{b}^{u}+2 H \check{c}^{u}\right) V_{1}^{0.5}(y)+\left(2 H \check{b}^{u}\right. \\
& \left.\left.+2 H \check{c}^{u}\right) V_{1}^{1.5}(y)+\frac{\kappa}{\varrho}\right\} d t-\varrho e^{\kappa t}\left(1+V_{1}(y)\right)^{\varrho-1} \frac{2}{W^{3}}\left(\sigma_{1}(t) y_{1}(t) d B_{1}(t)\right. \\
& \left.+\sigma_{2}(t) y_{2}(t) d B_{2}(t)\right) \\
\leq & e^{\kappa t} \Lambda(y) d t-\varrho e^{\kappa t}\left(1+V_{1}(y)\right)^{\varrho-1} \frac{2}{W^{3}}\left(\sigma_{1}(t) y_{1}(t) d B_{1}(t)\right. \\
& \left.+\sigma_{2}(t) y_{2}(t) d B_{2}(t)\right),
\end{aligned}
$$

where

$$
\begin{aligned}
\Lambda(y)= & \varrho\left(1+V_{1}(y)\right)^{\varrho-2}\left\{-2 V_{1}^{2}(y)\left[\frac{\hat{\mathcal{R}}^{l}-\varrho\left(\check{\sigma}^{u}\right)^{2}}{4}-\frac{0.5 \kappa}{\varrho}\right]+\left[3\left(\check{\sigma}^{u}\right)^{2}+\frac{2 \kappa}{\varrho}\right] V_{1}(y)\right. \\
& \left.+\left(2 H \check{b}^{u}+2 H \check{c}^{u}\right) V_{1}^{0.5}(y)+\left(2 H \check{b}^{u}+2 H \check{c}^{u}\right) V_{1}^{1.5}(y)+\frac{\kappa}{\varrho}\right\}
\end{aligned}
$$


Note that (3.10), we see that

$$
\left(2 H \check{b}^{u}+2 H \check{c}^{u}\right) V_{1}^{0.5}(y)+\left(2 H \check{b}^{u}+2 H \check{c}^{u}\right) V_{1}^{1.5}(y)=\frac{2 H \check{b}^{u}+2 H \check{c}^{u}}{W}+\frac{2 H \check{b}^{u}+2 H \check{c}^{u}}{W^{3}}
$$

is an upper bounded for $W>0$, denoted by $\mathscr{L}$.

Set

$$
P_{1}=2\left[\frac{\hat{\mathcal{R}}^{l}-\varrho\left(\check{\sigma}^{u}\right)^{2}}{4}-\frac{0.5 \kappa}{\varrho}\right], \quad P_{2}=3\left(\check{\sigma}^{u}\right)^{2}+\frac{2 \kappa}{\varrho}, \quad P_{3}=\frac{\kappa}{\varrho}+\mathscr{L} .
$$

Obviously, one sees from (3.13) that $P_{i}>0, i=1,2,3$.

It follows from (3.16) and (3.17) that

$$
\Lambda(y)=\varrho\left(1+V_{1}(y)\right)^{\varrho-2}\left\{-P_{1} V_{1}^{2}(y)+P_{2} V_{1}(y)+P_{3}\right\}=\varrho\left(1+V_{1}(y)\right)^{\varrho-2} \Lambda_{1}(y),
$$

where

$$
\Lambda_{1}(y)=-P_{1} V_{1}^{2}(y)+P_{2} V_{1}(y)+P_{3} .
$$

Denote

$$
K_{1}=\frac{P_{2}+\sqrt{P_{2}^{2}+4 P_{1} P_{3}}}{2 P_{1}}, \quad K_{2}=\frac{P_{2}^{2}+4 P_{1} P_{3}}{4 P_{1}}, \quad K_{3}=\max \left\{\varrho\left(1+K_{1}\right)^{\varrho-2}, \varrho\right\} .
$$

Similar to the proof of Theorem 3.1 in [8], two cases are considered (i.e., $0<V_{1}(y) \leq K_{1}$ and $\left.V_{1}(y) \geq K_{1}\right)$ and we see that $\Lambda(y)$ is an upper bounded, denoted by $\Lambda^{*}=K_{2} K_{3}$. Therefore, from (3.15) it follows that

$$
d V_{3}(y) \leq \Lambda^{*} e^{\kappa t} d t-\varrho e^{\kappa t}\left(1+V_{1}(y)\right)^{\varrho-1} \frac{2}{W^{3}}\left(\sigma_{1}(t) y_{1}(t) d B_{1}(t)+\sigma_{2}(t) y_{2}(t) d B_{2}(t)\right) .
$$

Integrating and taking expectation on both sides of (3.20) gives us that

$$
E\left[V_{3}(y)\right]=E\left[e^{\kappa t}\left(1+V_{1}(y)\right)^{\varrho}\right] \leq\left(1+V_{1}(y(0))\right)^{\varrho}+\frac{\Lambda^{*}}{\kappa} e^{\kappa t},
$$

which implies that

$$
\limsup _{t \rightarrow+\infty} E\left[\left(y_{1}(t)+y_{2}(t)\right)^{-2 \varrho}\right] \leq \limsup _{t \rightarrow+\infty} E\left[\left(1+V_{1}(y)\right)^{\varrho}\right] \leq \frac{\Lambda^{*}}{\kappa} .
$$

Hence

$$
\begin{aligned}
& \limsup _{t \rightarrow+\infty} E\left[\left(x_{1}(t)+x_{2}(t)\right)^{-2 \varrho}\right] \\
& \quad=\limsup _{t \rightarrow+\infty} E\left[\left(\prod_{0<t_{k}<t}\left(1+\mathcal{H}_{1 k}\right) y_{1}(t)+\prod_{0<t_{k}<t}\left(1+\mathcal{H}_{2 k}\right) y_{2}(t)\right)^{-2 \varrho}\right] \\
& \quad \leq h^{-2 \varrho} \frac{\Lambda^{*}}{\kappa}
\end{aligned}
$$


where $h=\min \left\{h_{1}, h_{2}\right\}, h_{i}$ is defined in (1.1). Thus

$$
\limsup _{t \rightarrow+\infty} E\left(|x(t)|^{-2 \varrho}\right) \leq \limsup _{t \rightarrow+\infty} E\left[\frac{1}{4}\left(x_{1}(t)+x_{2}(t)\right)^{2}\right]^{-\varrho}=4^{\varrho} h^{-2 \varrho} \frac{\Lambda^{*}}{\kappa}=\mathscr{F}
$$

For any $\varepsilon>0$, we let $\alpha=\left(\frac{\varepsilon}{\mathscr{F}}\right)^{1 / 2 \varrho}$. Applying Chebyshev's inequality leads to

$$
\mathbb{P}\{|x(t)|<\alpha\}=\mathbb{P}\left\{|x(t)|^{-2 \varrho}>\alpha^{-2 \varrho}\right\} \leq \alpha^{2 \varrho} E\left(|x(t)|^{-2 \varrho}\right) .
$$

So

$$
\limsup _{t \rightarrow+\infty} \mathbb{P}\{|x(t)|<\alpha\} \leq \alpha^{2 \varrho} \mathscr{F}=\varepsilon
$$

namely,

$$
\liminf _{t \rightarrow+\infty} \mathbb{P}\{|x(t)| \geq \alpha\} \geq 1-\varepsilon
$$

Now let us prove that, for arbitrary $\varepsilon>0$, there exists a constant $\beta>0$ such that $\liminf _{t \rightarrow+\infty} \mathbb{P}\{|x(t)| \leq \beta\} \geq 1-\varepsilon$. For $p>1$ arbitrarily, set

$$
V_{4}(y)=y_{1}^{p}+y_{2}^{p}
$$

Using Itô's formula to (2.1) yields

$$
\begin{aligned}
d V_{4}(y)= & p y_{1}^{p}(t)\left[a_{1}(t)-b_{1}(t) \prod_{0<t_{k}<t}\left(1+\mathcal{H}_{1 k}\right) y_{1}(t)-\frac{c_{2}(t) \prod_{0<t_{k}<t}\left(1+\mathcal{H}_{2 k}\right) y_{2}(t)}{1+\prod_{0<t_{k}<t}\left(1+\mathcal{H}_{2 k}\right) y_{2}(t)}\right. \\
& \left.+0.5(p-1) \sigma_{1}^{2}(t)\right] d t+p \sigma_{1}(t) y_{1}^{p}(t) d B_{1}(t) \\
& +p y_{2}^{p}(t)\left[a_{2}(t)-b_{2}(t) \prod_{0<t_{k}<t}\left(1+\mathcal{H}_{2 k}\right) y_{2}(t)-\frac{c_{1}(t) \prod_{0<t_{k}<t}\left(1+\mathcal{H}_{1 k}\right) y_{1}(t)}{1+\prod_{0<t_{k}<t}\left(1+\mathcal{H}_{1 k}\right) y_{1}(t)}\right. \\
& \left.+0.5(p-1) \sigma_{2}^{2}(t)\right] d t+p \sigma_{2}(t) y_{2}^{p}(t) d B_{2}(t) \\
\leq & p y_{1}^{p}(t)\left[a_{1}^{u}-b_{1}^{l} h_{1} y_{1}(t)+0.5(p-1)\left(\sigma_{1}^{u}\right)^{2}\right] d t+p \sigma_{1}(t) y_{1}^{p}(t) d B_{1}(t) \\
& +p y_{2}^{p}(t)\left[a_{2}^{u}-b_{2}^{l} h_{2} y_{2}(t)+0.5(p-1)\left(\sigma_{2}^{u}\right)^{2}\right] d t+p \sigma_{2}(t) y_{2}^{p}(t) d B_{2}(t) .
\end{aligned}
$$

An application of Itô's formula again gives

$$
\begin{aligned}
d\left[e^{t} V_{4}(y)\right]= & e^{t} V_{4}(y) d t+e^{t} d V_{4}(y) \\
\leq & e^{t} p y_{1}^{p}(t)\left[1 / p+a_{1}^{u}-b_{1}^{l} h_{1} y_{1}(t)+0.5(p-1)\left(\sigma_{1}^{u}\right)^{2}\right] d t \\
& +p e^{t} \sigma_{1}(t) y_{1}^{p}(t) d B_{1}(t) \\
& +e^{t} p y_{2}^{p}(t)\left[1 / p+a_{2}^{u}-b_{2}^{l} h_{2} y_{2}(t)+0.5(p-1)\left(\sigma_{2}^{u}\right)^{2}\right] d t \\
& +p e^{t} \sigma_{2}(t) y_{2}^{p}(t) d B_{2}(t) .
\end{aligned}
$$


Note that $p \sum_{i=1}^{2} y_{i}^{p}(t)\left[1 / p+a_{i}^{u}-b_{i}^{l} h_{i} y_{i}(t)+0.5(p-1)\left(\sigma_{i}^{u}\right)^{2}\right]$ has a maximum, where we denote

$$
\Pi_{1}(p)=\sum_{i=1}^{2} \frac{p^{p+1}\left[1 / p+a_{i}^{u}+0.5(p-1)\left(\sigma_{i}^{u}\right)^{2}\right]^{p+1}}{(p+1)^{p+1}\left(b_{i}^{l} h_{i}\right)^{p}}
$$

The inequality (3.25) then can be rewritten as

$$
d\left[e^{t} V_{4}(y)\right] \leq e^{t} \Pi_{1}(p) d t+p e^{t} \sigma_{1}(t) y_{1}^{p}(t) d B_{1}(t)+p e^{t} \sigma_{2}(t) y_{2}^{p}(t) d B_{2}(t) .
$$

Integrating and taking the expectation on both sides of (3.26) give

$$
E\left[e^{t} V_{4}(y)\right] \leq V_{4}(y(0))+\left(e^{t}-1\right) \Pi_{1}(p),
$$

which implies that

$$
\limsup _{t \rightarrow+\infty} E\left(y_{1}^{p}+y_{2}^{p}\right) \leq \Pi_{1}(p)
$$

In other words, one has shown that

$$
\begin{aligned}
\limsup _{t \rightarrow+\infty} E\left(x_{1}^{p}+x_{2}^{p}\right) & =\limsup _{t \rightarrow+\infty} E\left(\left[\prod_{0<t_{k}<t}\left(1+\mathcal{H}_{1 k}\right)\right]^{p} y_{1}^{p}(t)+\left[\prod_{0<t_{k}<t}\left(1+\mathcal{H}_{2 k}\right)\right]^{p} y_{2}^{p}(t)\right) \\
& \leq H^{p} \Pi_{1}(p),
\end{aligned}
$$

where $H=\max \left\{H_{1}, H_{2}\right\}, H_{i}$ is defined in (1.1). Let $\delta=\left(\frac{2^{p-1} H^{p} \Pi_{1}(p)}{\varepsilon}\right)^{1 / p}$. One derives, by Chebyshev's inequality, that

$$
\mathbb{P}\{|x(t)|>\delta\}=\mathbb{P}\left\{|x(t)|^{p}>\delta^{p}\right\} \leq \frac{E\left(|x(t)|^{p}\right)}{\delta^{p}} \leq \frac{E\left(x_{1}(t)+x_{2}(t)\right)^{p}}{\delta^{p}} \leq \frac{2^{p-1} E\left(x_{1}^{p}(t)+x_{2}^{p}(t)\right)}{\delta^{p}},
$$

which shows that

$$
\limsup _{t \rightarrow+\infty} \mathbb{P}\{|x(t)|>\delta\} \leq \frac{2^{p-1} H^{p} \Pi_{1}(p)}{\delta^{p}}=\varepsilon
$$

Therefore,

$$
\liminf _{t \rightarrow+\infty} \mathbb{P}\{|x(t)| \leq \delta\} \geq 1-\varepsilon
$$

which together with (3.22), shows that system (1.2) is stochastically permanent. One completes the proof of Theorem 3.2.

Remark 3.1 Theorems 3.1 and 3.2 show that the large intensities of noises lead to the extinction of both species while system (1.2) is stochastically permanent provided that the intensities of noises are suitable small. 


\section{Asymptotic properties}

In this section, we will estimate the limit of the average in time of the sample paths of every component of the solution.

Assign

$$
\mathcal{Q}_{i}=\liminf _{t \rightarrow+\infty} t^{-1}\left(\sum_{0<t_{k}<t} \ln \left(1+\mathcal{H}_{i k}\right)+\int_{0}^{t} \mathcal{R}_{i}(s) d s\right), \quad i=1,2
$$

Theorem 4.1 The solution $\left(x_{1}(t), x_{2}(t)\right)$ of system (1.2) satisfies

$$
\limsup _{t \rightarrow+\infty} t^{-1} \int_{0}^{t} x_{i}(s) d s \leq \frac{\mathcal{R}_{i}^{*}}{b_{i}^{l}} \quad \text { a.s. }
$$

If $\mathcal{Q}_{i}>c_{j}^{u}$, then

$$
\liminf _{t \rightarrow+\infty} t^{-1} \int_{0}^{t} x_{i}(s) d s \geq \frac{\mathcal{Q}_{i}-c_{j}^{u}}{b_{i}^{u}} \text { a.s. }
$$

Proof We first prove (4.2). Using Itô's formula to system (2.1), one has

$$
\begin{aligned}
d \ln y_{i}(t)= & {\left[a_{i}(t)-b_{i}(t) \prod_{0<t_{k}<t}\left(1+\mathcal{H}_{i k}\right) y_{i}(t)-\frac{c_{j}(t) \prod_{0<t_{k}<t}\left(1+\mathcal{H}_{j k}\right) y_{j}(t)}{1+\prod_{0<t_{k}<t}\left(1+\mathcal{H}_{j k}\right) y_{j}(t)}\right.} \\
& \left.-0.5 \sigma_{i}^{2}(t)\right] d t+\sigma_{i}(t) d B_{i}(t) \\
= & {\left[\mathcal{R}_{i}(t)-b_{i}(t) x_{i}(t)-\frac{c_{j}(t) x_{j}(t)}{1+x_{j}(t)}\right] d t+\sigma_{i}(t) d B_{i}(t) . }
\end{aligned}
$$

Integrating on both sides of (4.4) shows that

$$
\ln y_{i}(t)-\ln y_{i}(0)=\int_{0}^{t} \mathcal{R}_{i}(s) d s-\int_{0}^{t} b_{i}(s) x_{i}(s) d s-\int_{0}^{t} \frac{c_{j}(s) x_{j}(s)}{1+x_{j}(s)} d s+\mathscr{M}_{i}(t),
$$

where $\mathscr{M}_{i}(t)$ is defined in (3.5). Similar to (3.6) and (3.7), we obtain

$$
\left\langle\mathscr{M}_{i}(t), \mathscr{M}_{i}(t)\right\rangle=\int_{0}^{t} \sigma_{i}^{2}(s) d s \leq\left(\sigma_{i}^{u}\right)^{2} t
$$

and

$$
\lim _{t \rightarrow+\infty} \frac{\mathscr{M}_{i}(t)}{t}=0 \quad \text { a.s. }
$$

It follows from (4.5) that

$$
\begin{aligned}
& \sum_{0<t_{k}<t} \ln \left(1+\mathcal{H}_{i k}\right)+\ln y_{i}(t)-\ln y_{i}(0) \\
& \quad=\sum_{0<t_{k}<t} \ln \left(1+\mathcal{H}_{i k}\right)+\int_{0}^{t} \mathcal{R}_{i}(s) d s-\int_{0}^{t} b_{i}(s) x_{i}(s) d s-\int_{0}^{t} \frac{c_{j}(s) x_{j}(s)}{1+x_{j}(s)} d s+\mathscr{M}_{i}(t) .
\end{aligned}
$$


Rewriting (4.8), we have

$$
\begin{aligned}
\ln x_{i}(t)-\ln x_{i}(0)= & \sum_{0<t_{k}<t} \ln \left(1+\mathcal{H}_{i k}\right)+\int_{0}^{t} \mathcal{R}_{i}(s) d s-\int_{0}^{t} b_{i}(s) x_{i}(s) d s \\
& -\int_{0}^{t} \frac{c_{j}(s) x_{j}(s)}{1+x_{j}(s)} d s+\mathscr{M}_{i}(t) .
\end{aligned}
$$

On the other hand, for arbitrarily fixed $\varepsilon>0$, there is a positive constant $T$ such that $t \geq T$

$$
\frac{\ln x(0)}{t} \leq \frac{\varepsilon}{3}, t^{-1}\left(\sum_{0<t_{k}<t} \ln \left(1+\mathcal{H}_{i k}\right)+\int_{0}^{t} \mathcal{R}_{i}(s) d s\right) \leq \mathcal{R}_{i}^{*}+\frac{\varepsilon}{3}, \quad \frac{\mathscr{M}_{i}(t)}{t} \leq \frac{\varepsilon}{3}
$$

Substituting (4.10) into (4.9) yields

$$
\ln x_{i}(t) \leq\left(\mathcal{R}_{i}^{*}+\varepsilon\right) t-\int_{0}^{t} b_{i}(s) x_{i}(s) d s \leq \varphi_{i} t-b_{i}^{l} \int_{0}^{t} x_{i}(s) d s,
$$

where $\varphi_{i}=\mathcal{R}_{i}^{*}+\varepsilon$. Denote

$$
g_{i}(t)=\int_{0}^{t} x_{i}(s) d s
$$

then we have

$$
\exp \left\{b_{i}^{l} g_{i}(t)\right\} \frac{d g_{i}(t)}{d t} \leq \exp \left\{\varphi_{i} t\right\}
$$

Integrating from $T$ to $t$ on both sides of (4.12) shows that

$$
\exp \left\{b_{i}^{l} g_{i}(t)\right\} \leq \exp \left\{b_{i}^{l} g_{i}(T)\right\}+b_{i}^{l} \varphi_{i}^{-1} \exp \left\{\varphi_{i} t\right\}-b_{i}^{l} \varphi_{i}^{-1} \exp \left\{\varphi_{i} T\right\}
$$

Taking the logarithm on both sides of (4.13), one finds that

$$
g_{i}(t) \leq\left(b_{i}^{l}\right)^{-1} \ln \left\{b_{i}^{l} \varphi_{i}^{-1} \exp \left(\varphi_{i} t\right)+\exp \left\{b_{i}^{l} g_{i}(T)\right\}-b_{i}^{l} \varphi_{i}^{-1} \exp \left(\varphi_{i} T\right)\right\}
$$

Therefore,

$$
\begin{aligned}
\limsup _{t \rightarrow+\infty} t^{-1} \int_{0}^{t} x_{i}(s) d s \leq & \left(b_{i}^{l}\right)^{-1} \limsup _{t \rightarrow+\infty} t^{-1} \ln \left[b_{i}^{l} \varphi_{i}^{-1} \exp \left(\varphi_{i} t\right)\right. \\
& \left.+\exp \left\{b_{i}^{l} g_{i}(T)\right\}-b_{i}^{l} \varphi_{i}^{-1} \exp \left(\varphi_{i} T\right)\right] .
\end{aligned}
$$

By the L'Hospital's rule,

$$
\limsup _{t \rightarrow+\infty} t^{-1} \int_{0}^{t} x_{i}(s) d s \leq\left(b_{i}^{l}\right)^{-1} \limsup _{t \rightarrow+\infty} t^{-1} \ln \left[b_{i}^{l} \varphi_{i}^{-1} \exp \left(\varphi_{i} t\right)\right]=\frac{\varphi_{i}}{b_{i}^{l}}
$$

The required assertion follows from the arbitrariness of $\varepsilon$. 
In the sequel, we show that (4.3) holds. For arbitrarily fixed $\varepsilon>0$, there is a constant $\bar{T}$ such that

$$
\frac{\ln x(0)}{t} \geq-\frac{\varepsilon}{3}, \frac{\sum_{0<t_{k}<t} \ln \left(1+\mathcal{H}_{i k}\right)+\int_{0}^{t} \mathcal{R}_{i}(s) d s}{t} \geq \mathcal{Q}_{i}-\frac{\varepsilon}{3}, \quad \frac{\mathscr{M}(t)}{t} \geq-\frac{\varepsilon}{3}
$$

for all $t \geq \bar{T}$. Substituting (4.17) into (4.9) gives

$$
\ln x_{i}(t) \geq\left(\mathcal{Q}_{i}-c_{j}^{u}-\varepsilon\right) t-b_{i}^{u} \int_{0}^{t} x_{i}(s) d s=\mathcal{L}_{i} t-b_{i}^{u} \int_{0}^{t} x_{i}(s) d s,
$$

where $\mathcal{L}_{i}=\mathcal{Q}_{i}-c_{j}^{u}-\varepsilon$. Then it can be shown that

$$
\ln \left(\frac{d g_{i}(t)}{d t}\right) \geq \mathcal{L}_{i} t-b_{i}^{u} g_{i}(t)
$$

Integrating (4.19) from $\bar{T}$ to $t$ leads to

$$
\exp \left(b_{i}^{u} g_{i}(t)\right) \geq b_{i}^{u} \mathcal{L}_{i}^{-1} \exp \left(\mathcal{L}_{i} t\right)+\exp \left(b_{i}^{u} g_{i}(\bar{T})\right)-b_{i}^{u} \mathcal{L}_{i}^{-1} \exp \left(\mathcal{L}_{i} \bar{T}\right) .
$$

Taking the logarithm on both sides of (4.20) gives

$$
g_{i}(t) \geq\left(b_{i}^{u}\right)^{-1} \ln \left\{b_{i}^{u} \mathcal{L}_{i}^{-1} \exp \left(\mathcal{L}_{i} t\right)+\exp \left(b_{i}^{u} g_{i}(\bar{T})\right)-b_{i}^{u} \mathcal{L}_{i}^{-1} \exp \left(\mathcal{L}_{i} \bar{T}\right)\right\} .
$$

We therefore obtain

$$
\begin{aligned}
& \liminf _{t \rightarrow+\infty} t^{-1} \int_{0}^{t} x_{i}(s) d s \\
& \quad \geq\left(b_{i}^{u}\right)^{-1} \liminf _{t \rightarrow+\infty} t^{-1} \ln \left\{b_{i}^{u} \mathcal{L}_{i}^{-1} \exp \left(\mathcal{L}_{i} t\right)+\exp \left(b_{i}^{u} g_{i}(\bar{T})\right)-b_{i}^{u} \mathcal{L}_{i}^{-1} \exp \left(\mathcal{L}_{i} \bar{T}\right)\right\} .
\end{aligned}
$$

According to the L'Hospital's rule, one derives that

$$
\liminf _{t \rightarrow+\infty} t^{-1} \int_{0}^{t} x_{i}(s) d s \geq\left(b_{i}^{u}\right)^{-1} \liminf _{t \rightarrow+\infty} t^{-1} \ln \left\{b_{i}^{u} \mathcal{L}_{i}^{-1} \exp \left(\mathcal{L}_{i} t\right)\right\}=\frac{\mathcal{L}_{i}}{b_{i}^{u}}
$$

The desired assertion immediately follows from the arbitrariness of $\varepsilon$. The proof of Theorem 4.1 is completed.

Remark 4.1 In Theorem 4.1, $\mathcal{Q}_{i}>c_{j}^{u}$ may be interpreted as saying that the intrinsic growth rate of species $i$ is large while the intensity of noise for species $i$ and the interspecific competitive rate of species $j$ are small.

\section{Global attractivity}

This section is concerned with the global attractivity of system (1.2), and we first give Lemma 5.1.

Lemma 5.1 Suppose that $y(t)$ is a solution of system (2.1) with initial value $y(0)>0$, then almost every sample path of $y_{i}(t)$ is uniformly continuous for $t \geq 0$. 
Proof It follows from (3.27) that there exists $T^{*}>0$ such that, for all $t \geq T^{*}$,

$$
E\left(y_{1}^{p}(t)+y_{2}^{p}(t)\right) \leq \Pi_{1}(p) .
$$

Also, by the continuity of $E\left(y_{1}^{p}(t)+y_{2}^{p}(t)\right)$, there exists a $\Pi_{2}(p)$ such that, for $t \leq T^{*}$,

$$
E\left(y_{1}^{p}(t)+y_{2}^{p}(t)\right) \leq \Pi_{2}(p) .
$$

Set $\Pi(p)=\max \left\{\Pi_{1}(p), \Pi_{2}(p)\right\}$, it follows from (5.1) and (5.2) that, for all $t \geq 0$,

$$
E\left(y_{1}^{p}(t)+y_{2}^{p}(t)\right) \leq \Pi(p) .
$$

The rest of proof is similar to Lemma 7 in Ref. [5]; we omit it.

\section{Assign}

$$
b_{1}^{l} b_{2}^{l}>c_{1}^{u} c_{2}^{u}
$$

It is not difficult to verify that there exists a pair of positive constants $\eta_{1}$ and $\eta_{2}$ such that

$$
\eta_{1} b_{1}^{l}-\eta_{2} c_{1}^{u}=\epsilon, \quad \eta_{2} b_{2}^{l}-\eta_{1} c_{2}^{u}=\epsilon
$$

where $\epsilon>0$ is fixed.

Theorem 5.1 If $b_{1}^{l} b_{2}^{l}>c_{1}^{u} c_{2}^{u}$, then system (1.2) is globally attractive.

Proof Let $x(t)$ and $x^{*}(t)$ be two solutions of system (1.2) with initial values $x(0)>0$ and $x^{*}(0)>0$, respectively. Suppose that $x(t)$ is a solution of the following system with $y(0)=$ $x(0)$ :

$$
\left\{\begin{aligned}
d y_{1}(t)= & y_{1}(t)\left[a_{1}(t)-b_{1}(t) \prod_{0<t_{k}<t}\left(1+\mathcal{H}_{1 k}\right) y_{1}(t)-\frac{c_{2}(t) \prod_{0<t_{k}<t}\left(1+\mathcal{H}_{2 k}\right) y_{2}(t)}{1+\prod_{0<t_{k}<t}\left(1+\mathcal{H}_{2 k}\right) y_{2}(t)}\right] d t \\
& +\sigma_{1}(t) y_{1}(t) d B_{1}(t), \\
d y_{2}(t)= & y_{2}(t)\left[a_{2}(t)-b_{2}(t) \prod_{0<t_{k}<t}\left(1+\mathcal{H}_{2 k}\right) y_{2}(t)-\frac{c_{1}(t) \prod_{0<t_{k}<t}\left(1+\mathcal{H}_{1 k}\right) y_{1}(t)}{1+\prod_{0<t_{k}<t}\left(1+\mathcal{H}_{1 k}\right) y_{1}(t)}\right] d t \\
& +\sigma_{2}(t) y_{2}(t) d B_{2}(t),
\end{aligned}\right.
$$

and $x^{*}(t)$ is a solution of the following system with $y^{*}(0)=x^{*}(0)$ :

$$
\left\{\begin{aligned}
d y_{1}(t)= & y_{1}(t)\left[a_{1}(t)-b_{1}(t) \prod_{0<t_{k}<t}\left(1+\mathcal{H}_{1 k}\right) y_{1}(t)-\frac{c_{2}(t) \prod_{0<t_{k}<t}\left(1+\mathcal{H}_{2 k}\right) y_{2}(t)}{1+\prod_{0<t_{k}<t}\left(1+\mathcal{H}_{2 k}\right) y_{2}(t)}\right] d t \\
& +\sigma_{1}(t) y_{1}(t) d B_{1}(t), \\
d y_{2}(t)= & y_{2}(t)\left[a_{2}(t)-b_{2}(t) \prod_{0<t_{k}<t}\left(1+\mathcal{H}_{2 k}\right) y_{2}(t)-\frac{c_{1}(t) \prod_{0<t_{k}<t}\left(1+\mathcal{H}_{1 k}\right) y_{1}(t)}{1+\prod_{0<t_{k}<t}\left(1+\mathcal{H}_{1 k}\right) y_{1}(t)}\right] d t \\
& +\sigma_{2}(t) y_{2}(t) d B_{2}(t) .
\end{aligned}\right.
$$

Obviously, $x_{i}(t)=\prod_{0<t_{k}<t}\left(1+\mathcal{H}_{i k}\right) y_{i}(t), x_{i}^{*}(t)=\prod_{0<t_{k}<t}\left(1+\mathcal{H}_{i k}\right) y_{i}^{*}(t), i=1,2$.

Define

$$
V(t)=\eta_{1}\left|\ln y_{1}(t)-\ln y_{1}^{*}(t)\right|+\eta_{2}\left|\ln y_{2}(t)-\ln y_{2}^{*}(t)\right|,
$$


where $\eta_{1}$ and $\eta_{2}$ are defined in (5.5). Applying Itô's formula and computing the right differential $D^{+} V(t)$ yields

$$
\begin{aligned}
& D^{+} V(t)=\eta_{1} \operatorname{sgn}\left(y_{1}(t)-y_{1}^{*}(t)\right) d\left(\ln y_{1}(t)-\ln y_{1}^{*}(t)\right) \\
& +\eta_{2} \operatorname{sgn}\left(y_{2}(t)-y_{2}^{*}(t)\right) d\left(\ln y_{2}(t)-\ln y_{2}^{*}(t)\right) \\
& =\eta_{1} \operatorname{sgn}\left(y_{1}(t)-y_{1}^{*}(t)\right)\left\{-b_{1}(t) \prod_{0<t_{k}<t}\left(1+\mathcal{H}_{1 k}\right)\left(y_{1}(t)-y_{1}^{*}(t)\right)\right. \\
& \left.-\frac{c_{2}(t) \prod_{0<t_{k}<t}\left(1+\mathcal{H}_{2 k}\right)\left(y_{2}(t)-y_{2}^{*}(t)\right)}{\left(1+\prod_{0<t_{k}<t}\left(1+\mathcal{H}_{2 k}\right) y_{2}(t)\right)\left(1+\prod_{0<t_{k}<t}\left(1+\mathcal{H}_{2 k}\right) y_{2}^{*}(t)\right)}\right\} d t \\
& +\eta_{2} \operatorname{sgn}\left(y_{2}(t)-y_{2}^{*}(t)\right)\left\{-b_{2}(t) \prod_{0<t_{k}<t}\left(1+\mathcal{H}_{2 k}\right)\left(y_{2}(t)-y_{2}^{*}(t)\right)\right. \\
& \left.-\frac{c_{1}(t) \prod_{0<t_{k}<t}\left(1+\mathcal{H}_{1 k}\right)\left(y_{1}(t)-y_{1}^{*}(t)\right)}{\left(1+\prod_{0<t_{k}<t}\left(1+\mathcal{H}_{1 k}\right) y_{1}(t)\right)\left(1+\prod_{0<t_{k}<t}\left(1+\mathcal{H}_{1 k}\right) y_{1}^{*}(t)\right)}\right\} d t \\
& \leq\left\{-\eta_{1} a_{1}(t) \prod_{0<t_{k}<t}\left(1+\mathcal{H}_{1 k}\right)\left|y_{1}(t)-y_{1}^{*}(t)\right|\right. \\
& +\frac{\eta_{1} c_{2}(t) \prod_{0<t_{k}<t}\left(1+\mathcal{H}_{2 k}\right)\left|y_{2}(t)-y_{2}^{*}(t)\right|}{\left(1+\prod_{0<t_{k}<t}\left(1+\mathcal{H}_{2 k}\right) y_{2}(t)\right)\left(1+\prod_{0<t_{k}<t}\left(1+\mathcal{H}_{2 k}\right) y_{2}^{*}(t)\right)} \\
& -\eta_{2} a_{2}(t) \prod_{0<t_{k}<t}\left(1+\mathcal{H}_{2 k}\right)\left|y_{2}(t)-y_{2}^{*}(t)\right| \\
& \left.+\frac{\eta_{2} c_{1}(t) \prod_{0<t_{k}<t}\left(1+\mathcal{H}_{1 k}\right)\left|y_{1}(t)-y_{1}^{*}(t)\right|}{\left(1+\prod_{0<t_{k}<t}\left(1+\mathcal{H}_{1 k}\right) y_{1}(t)\right)\left(1+\prod_{0<t_{k}<t}\left(1+\mathcal{H}_{1 k}\right) y_{1}^{*}(t)\right)}\right\} d t \\
& \leq-\left(\eta_{1} b_{1}(t)-\eta_{2} c_{1}(t)\right) \prod_{0<t_{k}<t}\left(1+\mathcal{H}_{1 k}\right)\left|y_{1}(t)-y_{1}^{*}(t)\right| d t \\
& -\left(\eta_{2} b_{2}(t)-\eta_{1} c_{2}(t)\right) \prod_{0<t_{k}<t}\left(1+\mathcal{H}_{2 k}\right)\left|y_{2}(t)-y_{2}^{*}(t)\right| d t \\
& \leq-\left(\eta_{1} b_{1}^{l}-\eta_{2} c_{1}^{u}\right) h_{1}\left|y_{1}(t)-y_{1}^{*}(t)\right| d t-\left(\eta_{2} b_{2}^{l}-\eta_{1} c_{2}^{u}\right) h_{2}\left|y_{2}(t)-y_{2}^{*}(t)\right| d t \\
& =-\epsilon h_{1}\left|y_{1}(t)-y_{1}^{*}(t)\right| d t-\epsilon h_{2}\left|y_{2}(t)-y_{2}^{*}(t)\right| d t \text {, }
\end{aligned}
$$

where $h_{1}$ and $h_{2}$ are defined in system (1.1). Consequently,

$$
V(t)-V(0) \leq-\epsilon h_{1} \int_{0}^{t}\left|y_{1}(s)-y_{1}^{*}(s)\right| d s-\epsilon h_{2} \int_{0}^{t}\left|y_{2}(s)-y_{2}^{*}(s)\right| d s
$$

That is,

$$
V(t)+\epsilon h_{1} \int_{0}^{t}\left|y_{1}(s)-y_{1}^{*}(s)\right| d s+\epsilon h_{2} \int_{0}^{t}\left|y_{2}(s)-y_{2}^{*}(s)\right| d s \leq V(0)<+\infty .
$$

Note that $V(t) \geq 0$, it then follows that $\left|y_{1}(t)-y_{1}^{*}(t)\right|,\left|y_{2}(t)-y_{2}^{*}(t)\right| \in L^{1}[0,+\infty)$. Using Lemma 5.1 and Barbalat's lemma in Ref. [9], we have

$$
\lim _{t \rightarrow+\infty}\left|y_{1}(t)-y_{1}^{*}(t)\right|=0, \quad \lim _{t \rightarrow+\infty}\left|y_{2}(t)-y_{2}^{*}(t)\right|=0 \quad \text { a.s. }
$$


As a consequence,

$$
\begin{aligned}
\lim _{t \rightarrow+\infty}\left|x_{1}(t)-x_{1}^{*}(t)\right| & =\lim _{t \rightarrow+\infty} \prod_{0<t_{k}<t}\left(1+\mathcal{H}_{1 k}\right)\left|y_{1}(t)-y_{1}^{*}(t)\right| \\
& \leq H_{1} \lim _{t \rightarrow+\infty}\left|y_{1}(t)-y_{1}^{*}(t)\right|=0, \\
\lim _{t \rightarrow+\infty}\left|x_{2}(t)-x_{2}^{*}(t)\right| & =\lim _{t \rightarrow+\infty} \prod_{0<t_{k}<t}\left(1+\mathcal{H}_{2 k}\right)\left|y_{2}(t)-y_{2}^{*}(t)\right| \\
& \leq H_{2} \lim _{t \rightarrow+\infty}\left|y_{2}(t)-y_{2}^{*}(t)\right|=0 \quad \text { a.s. }
\end{aligned}
$$

The proof of Theorem 5.1 is completed.

Remark 5.1 The assumption of Theorem 5.1, that is, $b_{1}^{l} b_{2}^{l}>c_{1}^{u} c_{2}^{u}$, the conclusion of biological meaning may be interpreted by saying that the influence of the intraspecific competition is greater than that of the interspecific competition.

\section{Numerical simulations}

In this section, we will demonstrate several specific numerical examples to confirm our analytical results.

Example 1 (Extinction) Consider the system

$$
\left\{\begin{aligned}
d x_{1}(t)= & x_{1}(t)\left[0.2+0.1 \sin t-(0.3+0.1 \sin t) x_{1}(t)\right. \\
& \left.-\frac{(0.4+0.1 \sin t) x_{2}(t)}{1+x_{2}(t)}\right] d t+(\sqrt{0.7}+0.1 \sin t) x_{1}(t) d B_{1}(t), \\
d x_{2}(t)= & x_{2}(t)\left[0.15+0.05 \sin t-(0.4+0.1 \sin t) x_{2}(t)\right. \\
& \left.-\frac{(0.5+0.1 \sin t) x_{1}(t)}{1+x_{1}(t)}\right] d t+(\sqrt{0.8}+0.1 \sin t) x_{2}(t) d B_{2}(t),
\end{aligned}\right\} \quad t \neq t_{k}
$$

Let $x_{1}(0)=0.4, x_{2}(0)=0.5, t_{k}=k, \mathcal{H}_{1 k}=\mathcal{H}_{2 k}=e^{(-1)^{k+1} / k}-1$, then $1<\prod_{k=1}^{\infty}\left(1+\mathcal{H}_{i k}\right)<2$, $i=1,2$, and $\mathcal{R}_{1}^{*}=-0.1525<0, \mathcal{R}_{2}^{*}=-0.2525<0$. It follows from Theorem 3.1 that system (6.1) becomes extinct, inspecting Figs. 1 and 2 .

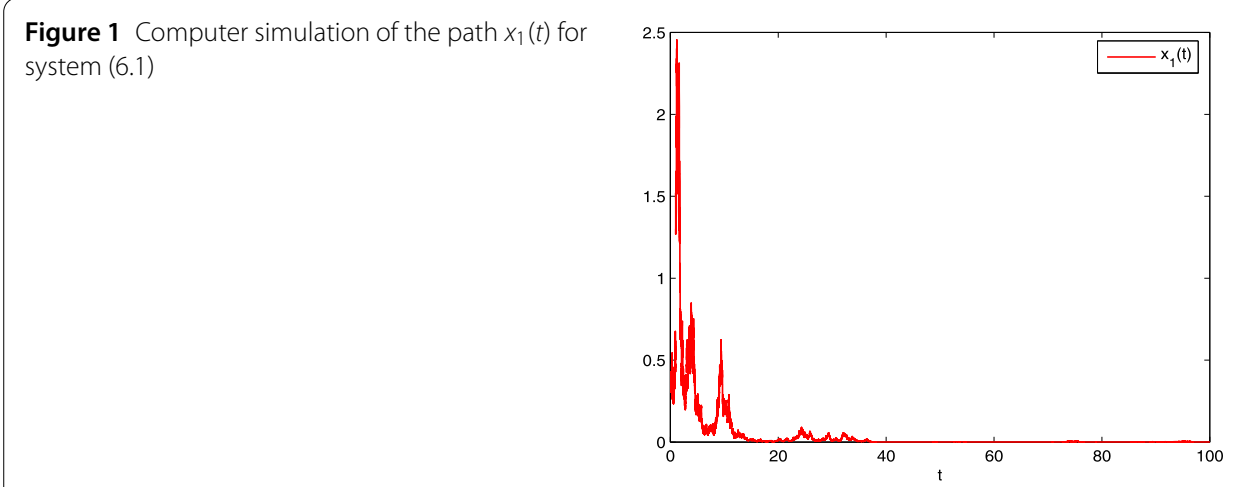


Figure 2 Computer simulation of the path $x_{2}(t)$ for system (6.1)

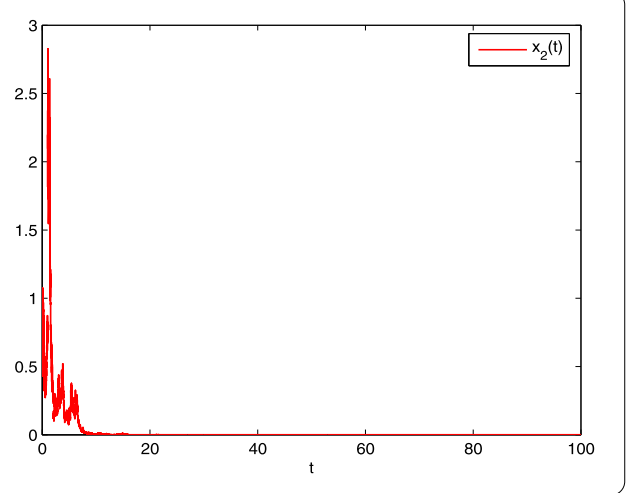

Figure 3 Computer simulations of $x_{1}(t)$ and $t^{-1} \int_{0}^{t} x_{1}(s) d s$ for system (6.2)

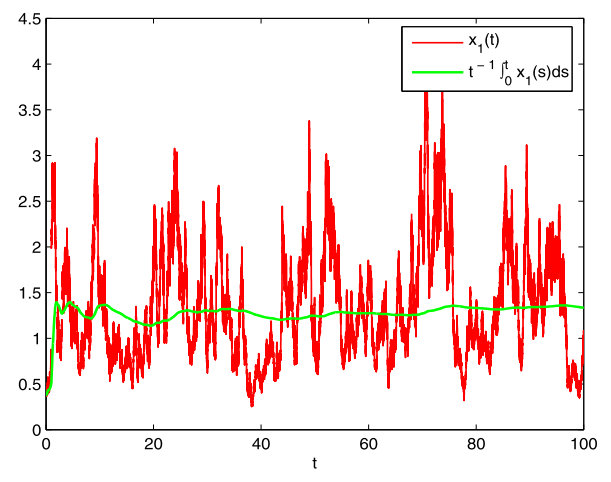

Figure 4 Computer simulations of $x_{2}(t)$ and $t^{-1} \int_{0}^{t} x_{2}(s) d s$ for system (6.2)

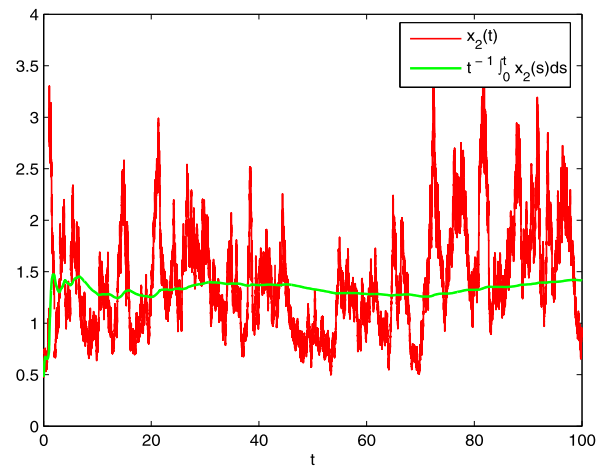

Example 2 (Stochastic permanence and asymptotic properties) Consider the system

$$
\left\{\begin{aligned}
d x_{1}(t)= & x_{1}(t)\left[0.65+0.05 \sin t-(0.3+0.1 \sin t) x_{1}(t)\right. \\
& \left.-\frac{(0.2+0.1 \sin t) x_{2}(t)}{1+x_{2}(t)}\right] d t+(\sqrt{0.2}+0.1 \sin t) x_{1}(t) d B_{1}(t), \quad \\
d x_{2}(t)= & x_{2}(t)\left[0.75+0.05 \sin t-(0.4+0.1 \sin t) x_{2}(t)\right. \\
& \left.-\frac{(0.15+0.05 \sin t) x_{1}(t)}{1+x_{1}(t)}\right] d t+(\sqrt{0.13}+0.1 \sin t) x_{2}(t) d B_{2}(t),
\end{aligned}\right\} \quad t \neq t_{k}
$$


Choose $x_{1}(0)=0.4, x_{2}(0)=0.5, t_{k}=k, \mathcal{H}_{1 k}=\mathcal{H}_{2 k}=e^{(-1)^{k+1} / k}-1$, then $1<\prod_{k=1}^{\infty}(1+$ $\left.\mathcal{H}_{i k}\right)<2, i=1,2$, and $\mathcal{R}_{1}^{l} \approx 0.4503, \mathcal{R}_{2}^{l} \approx 0.5939, \hat{\mathcal{R}}^{l} \approx 0.4503>0$. By Theorem 3.2 we know that system (6.2) is stochastically permanent. By Theorem 4.1, we obtain $\mathcal{Q}_{1}=0.5475>c_{2}^{u}=0.3, \mathcal{Q}_{2}=0.6825>c_{1}^{u}=0.2$ and so the solution of system (6.2) obeys $0.6187 \leq \liminf _{t \rightarrow+\infty} t^{-1} \int_{0}^{t} x_{1}(s) d s \leq \limsup _{t \rightarrow+\infty} t^{-1} \int_{0}^{t} x_{1}(s) d s \leq 2.75,0.965 \leq$ $\liminf _{t \rightarrow+\infty} t^{-1} \int_{0}^{t} x_{2}(s) d s \leq \limsup \operatorname{su}_{t \rightarrow+\infty} t^{-1} \int_{0}^{t} x_{2}(s) d s \leq 2.28$; see Figs. 3 and 4 .

Example 3 (Global attractivity) Consider the system

$$
\left\{\begin{aligned}
d x_{1}(t)= & x_{1}(t)\left[0.65+0.05 \sin t-(0.6+0.1 \sin t) x_{1}(t)\right. \\
& \left.-\frac{(0.2+0.1 \sin t) x_{2}(t)}{1+x_{2}(t)}\right] d t+(\sqrt{0.3}+0.1 \sin t) x_{1}(t) d B_{1}(t), \\
d x_{2}(t)= & x_{2}(t)\left[0.75+0.05 \sin t-(0.5+0.1 \sin t) x_{2}(t)\right. \\
& \left.-\frac{(0.3+0.1 \sin t) x_{1}(t)}{1+x_{1}(t)}\right] d t+(\sqrt{0.2}+0.1 \sin t) x_{2}(t) d B_{2}(t),
\end{aligned}\right\} \quad t \neq t_{k}
$$

Take $x(0)=(0.6,0.3)$ and $x^{*}(0)=(1.2,0.6), t_{k}=k, \mathcal{H}_{1 k}=\mathcal{H}_{2 k}=e^{(-1)^{k+1} / k^{2}}-1$, then $1<$ $\prod_{k=1}^{\infty}\left(1+\mathcal{H}_{i k}\right)<e, i=1,2$, and $b_{1}^{l} b_{2}^{l}=0.2>c_{1}^{u} c_{2}^{u}=0.12$. By Theorem 5.1 one concludes that system (6.3) is globally attractive, as shown in Figs. 5 and 6.

Figure 5 Computer simulation of the paths $x_{1}(t)$ and $x_{1}^{*}(t)$ for system (6.3)

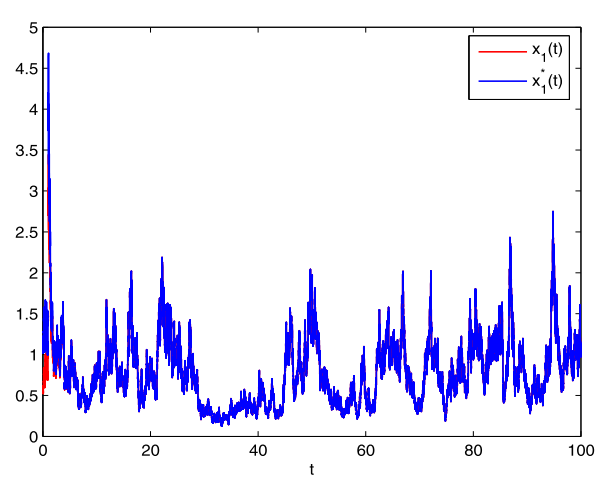

Figure 6 Computer simulation of the paths $x_{2}(t)$ and $x_{2}^{*}(t)$ for system (6.3)

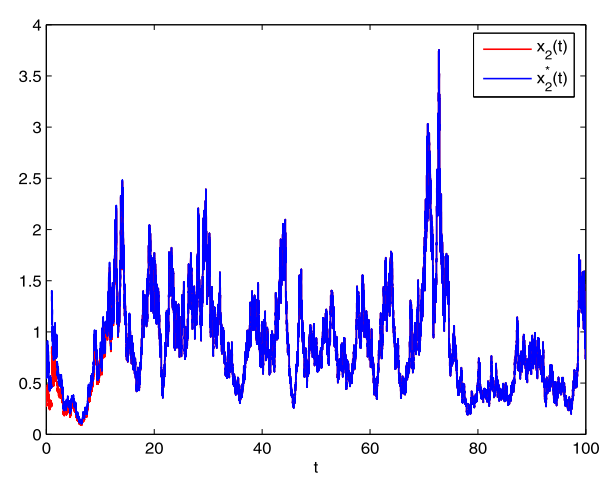




\section{Conclusions}

In this contribution, a novel nonautonomous impulsive stochastic two-species model with nonlinear interspecific competitive terms is established. The dynamics is analyzed in detail. It is shown that system (1.2) admits a unique global positive solution (i.e., no explosion in a finite time) for any given positive initial value. We also establish the sufficient conditions for extinction and stochastic permanence. Furthermore, the limit of the average in time of the sample paths of every component of the solution is estimated. Finally, the global attractivity is achieved. Our main results reveal that both species can go to extinction if the intensities of noises are large enough while system (1.2) can be stochastically permanent if the intensities of noises are relatively small in comparison with species intrinsic growth rates (see Theorems 3.1 and 3.2). In Theorem 4.1, $\mathcal{Q}_{i}>c_{j}^{u}$ may be interpreted as saying that the intrinsic growth rate of species $i$ is large while the intensity of noise for species $i$ and the interspecific competitive rate of species $j$ are small. It is also shown that the linear intraspecific competition is greater than the interspecific competition guaranteeing the global attractivity of system (1.2). We can find that the bounded impulses (i.e. the assumption in system (1.1) holds) have no influence on the above dynamic behaviors.

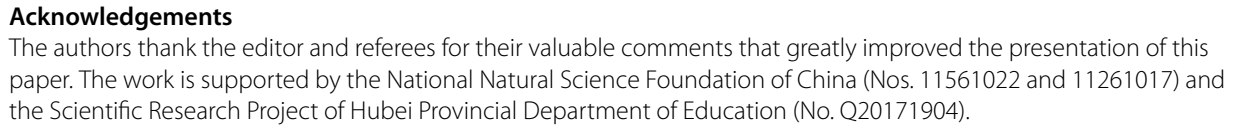

The authors thank the editor and referees for their valuable comments that greatly improved the presentation of this paper. The work is supported by the National Natural Science Foundation of China (Nos. 11561022 and 11261017) and the Scientific Research Project of Hubei Provincial Department of Education (No. Q20171904).

\section{Competing interests}

The authors declare that they have no competing interests.

Authors' contributions

Each of the authors, RT and SG et al. contributed to each part of this work equally and read and approved the final version of the manuscript.

\section{Author details}

${ }^{1}$ Department of Mathematics, Hubei University for Nationalities, Enshi, P.R. China. ${ }^{2}$ Department of Mathematics, Southern Illinois University, Carbondale, United States.

\section{Publisher's Note}

Springer Nature remains neutral with regard to jurisdictional claims in published maps and institutional affiliations.

Received: 15 August 2017 Accepted: 7 February 2018 Published online: 20 February 2018

\section{References}

1. Liu, Z.J., Wang, Q.L.: An almost periodic competitive system subject to impulsive perturbations. Appl. Math. Comput. 231, 377-385 (2014)

2. May, R.M.: Stability and Complexity in Model Ecosystems. Princeton University Press, Princeton (2001)

3. Chen, H.C., Ho, C.P.: Persistence and global stability on competition system with time-delay. Tunghai Sci. 5, 71-99 (2003)

4. Liu, Z.J., Tan, R.H., Chen, Y.P.: Modeling and analysis of a delayed competitive system with impulsive perturbations. Rocky Mt. J. Math. 38, 1505-1523 (2008)

5. Liu, M., Wang, K.: On a stochastic logistic equation with impulsive perturbations. Comput. Math. Appl. 63, 871-886 (2012)

6. Mao, X.R.: Stochastic Differential Equations and Applications. Horwood, Chichester (1997)

7. Bahar, A., Mao, X.R.: Stochastic delay Lotka-Volterra model. J. Math. Anal. Appl. 292, 364-380 (2004)

8. Tan, R.H., Liu, Z.J., Guo, S.L., Xiang, H.L.: On a nonautonomous competitive system subject to stochastic and impulsive perturbations. Appl. Math. Comput. 256, 702-714 (2015)

9. Barbalat, l.: Systemes d'équations differentielle d'oscillations nonlineaires. Rev. Roum. Math. Pures Appl. 4, 267-270 (1959) 